\title{
NIVEL DE CONOCIMIENTOS Y APTITUDES SOBRE COMPLICACIONES CRÓNICAS DE LA DIABETES MELLITUS 2, HOSPITAL NACIONAL PNP. LUIS N. SAENZ, 2016
}

\author{
LEVEL OF KNOWLEDGE AND SKILLS ON CHRONIC COMPLICATIONS OF DIABETES MELLITUS 2, HOSPITAL \\ NATIONAL PNP LUIS N. SAENZ, 2016 \\ Aldo Calderón-Rivera’
}

\begin{abstract}
RESUMEN
Objetivo: Determinar la relación entre conocimientos y aptitudes en pacientes diabéticos sobre las complicaciones crónicas de la diabetes mellitus 2 en el servicio de endocrinología del Hospital Nacional PNP Luis N. Suárez el 2016. Métodos: Estudio cuantitativo, descriptivo, transversal y correlacional. Se encuestaron a 110 pacientes diabéticos con un cuestionario para valorar los conocimientos (10 ítems con respuesta de selección múltiple) y otro para las aptitudes (tipo Lickert modificado). Los conocimientos se clasificaron como nivel alto, medio y bajo y las aptitudes como favorables, desfavorables e indiferentes según la Escala de Estanones. Se determinó la normalidad de las puntuaciones con la Prueba de Kolmogorov-Smirnov y las asociaciones con chi cuadrado, $\mathrm{p}<0.05$ y prueba de correlación de Pearson. Resultados: La edad media fue $49.85 \pm 6.97$ años, $74.5 \%$ sexo femenino, $75.5 \%$ con tiempo de enfermedad entre $1-5$ años, $67.3 \%$ con buena adherencia al tratamiento farmacológico. El nivel de conocimientos fue: alto $28.2 \%$, medio $49.1 \%$ y bajo $22.7 \%$; y las aptitudes fueron: favorables $23.6 \%$, desfavorables $58.2 \%$ e indiferentes $18.2 \%$. No existe relación entre conocimientos y aptitudes ( $p=0.247$ ). Sólo se encontró asociación estadística entre conocimiento y tratamiento farmacológico $(p<0.05)$. Conclusión: Los pacientes diabéticos tienen un nivel de conocimiento medio sobre sus complicaciones crónicas y sus aptitudes fueron predominantemente desfavorables. Es necesario realizar estrategias educativas para mejorar los conocimiento y las aptitudes de los diabéticos.
\end{abstract}

Palabras clave:Complicaciones crónicas; Diabetes mellitus 2; Conocimientos; Aptitudes; Correlación. (fuente: DeCS BIREME)

\begin{abstract}
Objective: To determine the relationship between knowledge and aptitude in diabetic patients regarding the chronic complications of diabetic mellitus 2 in the endocrinology service of National Hospital PNP Luis N. Saenz. Methods: Quantitative, descriptive, transverse and correlational study. 110 diabetic patients were surveyed with a questionnaire to assess the knowledge (10 items with a multiple choice response) and another one for the skills (modified Lickert type). The knowledge was classified as high, medium and low level and the skills as favorable, unfavorable and indifferent according to the Estanones Scale. The normality of the scores with the Kolmogorov-Smirnov test and associations with chi-square, $p<0.05$ and Pearson's correlation test were determined. Results: The mean age was $49.85 \pm 6.97$ years, $74.5 \%$ female, $75.5 \%$ with disease time between $1-5$ years, $67.3 \%$ with good adherence to pharmacological treatment. The level of knowledge was: high $28.2 \%$, medium $49.1 \%$ and low $22.7 \%$; And the skills were: favorable $23.6 \%$, unfavorable $58.2 \%$ and indifferent $18.2 \%$. There is no relation between knowledge and skills $(p=0.247)$. Only statistical association was found between knowledge and pharmacological treatment $(p<0.05)$. Conclusion: Diabetic patients have an average level of knowledge about their chronic complications and their abilities were predominantly unfavorable. It is necessary to carry out educational strategies to improve the knowledge and skills of diabetics.
\end{abstract}

Key words: Chronic complications; Diabetic mellitus 2; Knowledge; Aptitude; Correlation. (source: MeSH NLM)

'Hospital Nacional PNP "Luis N. Suárez", Lima-Perú.

Citar como: Aldo Calderón-Rivera. Nivel de conocimientos y aptitudes sobre complicaciones crónicas de la diabetes mellitus 2, Hospital Nacional PNP. Luis N. Saenz, 2016. [Artículo Original].2019;19(1):55-63. (Enero 2019). DOI 10.25176/RFMH.v19.n1.1793

( ) Los autores. Este artículo es publicado por la Revista de la Facultad de Medicina Humana, Universidad Ricardo Palma. Este es un artículo de Open Access distribuido bajo los términos de la Licencia Creative Commons Atribución-NoComercial-Compartirlgual 4.0 Internacional.(http://creativecommons.org/licenses/by-nc-sa/4.0/), que permite el uso no comercial, distribución y reproducción en cualquier medio, siempre que la obra original sea debidamente citadas. Para uso comercial, por favor póngase en contacto con revista.medicina@urp.pe 


\section{INTRODUCCIÓN}

En los últimos años, la diabetes mellitus 2 (DM2) se ha convertido en un flagelo para la humanidad por la elevada morbi-mortalidad asociada con esta enfermedad, que pese a los avances tecnológicos y terapéuticos aún no se ha logrado controlar los aspectos metabólicos involucrados, y de esta forma mejorar la calidad de vida y prolongar la sobrevida de estos pacientes ${ }^{1,2}$.

La DM2 es una enfermedad metabólica de aparición progresiva que se debe a la resistencia de la acción de la insulina sobre sus receptores celulares y/o a la disminución de su secreción pancreática lo que origina hiperglicemia, la cual, se depositará a nivel microvascular y será responsable de los cambios crónicos que padecerán posteriormente si la historia natural de la enfermedad no ha sido controlada ${ }^{3,4}$.

Los datos del MINSA no están actualizados ni completos, así, según los informes de la Oficina de Estadística e Informática del Ministerio de Salud, en el Perú, la DM2 afecta a casi 2 millones de personas y fue la décimo quinta causa de mortalidad en el Perú En el estudio PERUDIAB 2012 realizado por Seclén ${ }^{6}$ en una muestra representativa de 10 millones de adultos de 25 años que viven en nuestro país encontró una prevalencia de $7 \%$ de DM2 y $23 \%$ de hiperglicemia de ayuno considerado como pre diabetes.

Según el ENDES $2013^{7}$ realizada aproximadamente en 7000 mayores de 18 años a nivel nacional hay una prevalencia de sobrepeso de $33.8 \%$ y obesidad de $18.3 \%$, cifras alarmantes ya que, como se conoce, la DM2 es consecuencia de los trastornos metabólicos secundarios cuyo principal factor de riesgo son los malos hábitos alimenticios, a este factor también se puede sumar otros como antecedentes familiares de diabetes, hipertensión arterial, hipertrigliceridemia, adultos mayores y mujeres con diabetes gestacional ${ }^{2,6,8}$.

\section{MÉTODOS}

Se realizó un estudio de tipo observaciona, descriptivo analítico, de tipo transversal, correlacional. La muestra de estudio estuvo conformada por 110 pacientes diabéticos que cumplieron los criterios de inclusión y exclusión del estudio.

El análisis de los datos se realizó en el programa estadístico SPSS versión 22, a través de un análisis estadístico descriptivo (distribución porcentual de las características socio-epidemiológicas de los pacientes diabéticos estudiados y medidas de tendencia central y desviación estándar para la edad y tiempo de enfermedad). Para la elaboración de los gráficos se utilizó Microsoft Excel.

Para categorizar el nivel de conocimientos se obtuvo una calificación global de 0 a 10 puntos. Luego se realizó la Escala de Estanones de la siguiente manera:

- Escala de Estanones = Media+-0.75 (desviación estándar)

- Categoría superior (nivel de conocimientos alto): 4.6 $+0.75(1.6)=5.8:>5.8$

- Categoría intermedia (nivel de conocimientos medio): 3.4 - 5.8

- Categoría inferior: (nivel de conocimientos bajo): 4.6 $-0.75(1.6)=3.4:<3.4$

En las aptitudes se calificó cada respuesta según el rango de puntuación: 10-50 puntos.

Se clasificó a los pacientes según la puntuación obtenida siguiendo la escala de Estanones:

- Categoría superior (Favorables): $38.7+0.75(4.0)=$ $41.7:>41.7$

- Categoría intermedia (Desfavorables): 35.7 - 41.7

- Categoría inferior (Indiferentes): 38.7 - $0.75(4.0)=$ $35.7:<35.7$

De esta forma, se clasificó a cada sujeto de estudio en una de las tres categorías en ambos grupos, para finalmente presentarlos en una tabla con distribución porcentual.

La normalidad de las puntuaciones del nivel de conocimientos y aptitudes se determinó mediante la prueba de Kolmogorov-Smirnov.

Se obtuvo la prueba de correlación de Pearson para establecer la relación entre nivel de conocimientos y nivel de aptitudes sobre complicaciones crónicas de la DM2.

Durante el desarrollo de la investigación se actuó en base a los principios que manda la bioética, se guardó confidencialidad de sus datos y manteniéndolo en el anonimato. Se respetó los acápites de la declaración de Helsinki y se contó con la aprobación del comité de ética en investigación de la Facultad de Medicina de la Universidad Ricardo Palma.

\section{RESULTADOS}

Se encuestó a 110 pacientes diabéticos que se atendieron en el Servicio de Endocrinología del Hospital de la Policía Nacional del Perú Luis N. Sáenz 
durante el año 2016. La edad media fue 49.85 $\pm 6.97 \quad 2$ de 1-5 años, tratamiento farmacológico insulina años, el $42.7 \%$ con edad entre $50-59$ años, $74.5 \%$ fueron + hipoglicemiantes orales $44.5 \%$ y $67.3 \%$ con buena del sexo femenino, $56.4 \%$ con instrucción superior, adherencia al tratamiento farmacológico. (Tabla 1) $75.5 \%$ con tiempo de enfermedad de diabetes mellitus

Tabla 1. Características generales de los pacientes diabéticos encuestados en el servicio de endocrinología del hospital de la Policía Nacional del Perú "Luis N. Sáenz, 2016".

\section{CARACTERÍSTICA}

Grupos etáreos (años)

35-39

40-49

$50-59$

$60-69$

Sexo

Masculino

Femenino

Nivel de instrucción

Primaria

Secundaria

Superior

Tiempo de enfermedad de DM2

1-5 años

6-10 años

11-15 años

16-20 años

Tratamiento farmacológico para DM2

Insulina e hipoglicemiantes orales

Hipoglicemiantes orales

Insulina

Adherencia al tratamiento farmacológico de DM2

Buena

Mala

Total
62

6

47

74

36

82
24.5

32.7

\section{$\%$}

40.0

42.7

11.8

74.5

10.0

33.6

56.4

75.5

19.1

3.6

67.3

100.0 
Tabla 2. Prueba de Kolmogorov-Smirnov para valorar distribución de normalidad de las puntuaciones alcanzadas en el nivel de conocimientos y aptitudes de pacientes diabéticos.

\begin{tabular}{llcc}
\hline & & CONOCIMIENTOS & APTITUDES \\
\hline$N^{\circ}$ & & 110 & 110 \\
Parámetros & Media & 4,62 & 38,67 \\
normales & Desviación típica & 1,585 & 4,021 \\
& Absoluta & 0,134 & 0,160 \\
Diferencias más extremas & Positiva & 0,134 & 0,059 \\
& Negativa & $-0,113$ & $-0,160$ \\
Z de Kolmogorov-Smirnov & & 1,400 & 1,675 \\
Sig. asintót. (bilateral) & & 0,040 & 0,007 \\
\hline
\end{tabular}

a. La distribución de contraste es la Normal.

b. Se han calculado a partir de los datos.

Las puntuaciones obtenidas tras calificar las respuestas

Se encontró que el $49.1 \%$ tenía nivel medio en de los pacientes diabéticos en los cuestionarios para nivel de conocimientos y aptitudes, presentaron una distribución normal en la prueba de KolmogorovSmirnov. (Tabla 2)

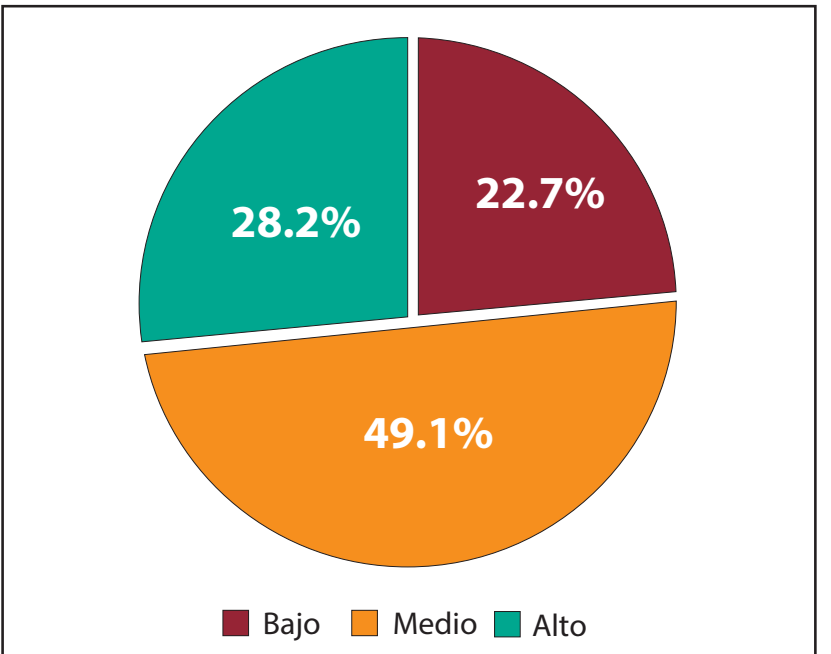

Gráfico 1. Nivel de conocimientos de los pacientes diabéticos sobre complicaciones crónicas de la diabetes mellitus 2. Servicio de Endocrinología del Hospital de la Policía Nacional del Perú Luis N. Sáenz, 2016.

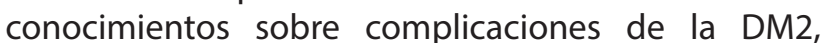
$28.2 \%$ nivel alto y $22.7 \%$ nivel bajo. (Figura 1 )

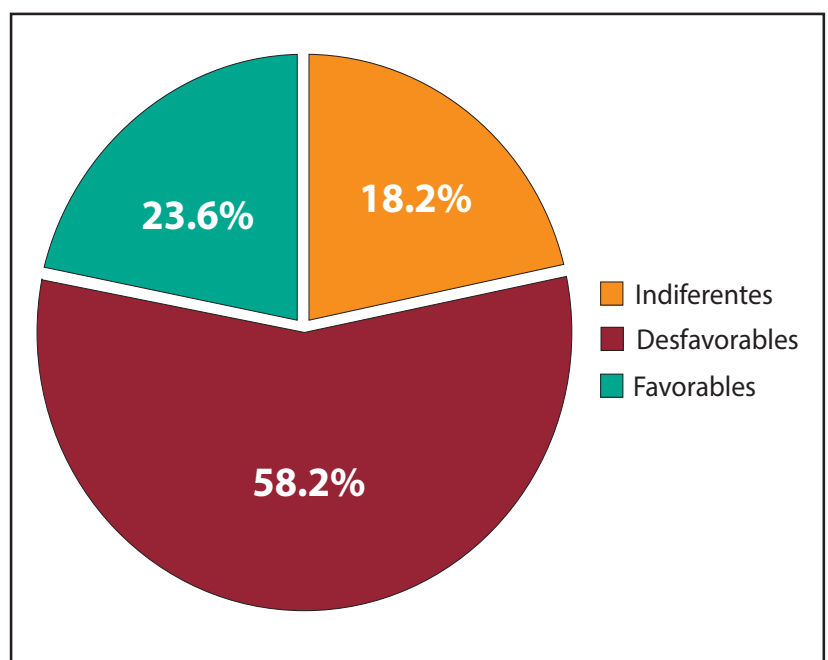

Gráfico 2. Aptitudes de los pacientes diabéticos sobre complicaciones crónicas de la diabetes mellitus 2 . Servicio de Endocrinología del Hospital de la Policía Nacional del Perú Luis N. Sáenz, 2016.

Se encontró que el $58.2 \%$ de los diabéticos encuestados tenían aptitudes desfavorables, 23.6\% aptitudes favorables y $18.2 \%$ aptitudes indiferentes. (Figura 2)

Tabla 3. Aptitudes y nivel de conocimientos sobre complicaciones crónicas de la DM2 en pacientes diabéticos. Servicio de Endocrinología del Hospital de la Policía Nacional del Perú Luis N. Sáenz, 2016.

\begin{tabular}{|c|c|c|c|c|c|c|c|}
\hline \multirow{3}{*}{$\begin{array}{c}\text { NIVEL DE } \\
\text { CONOCIMIENTOS }\end{array}$} & \multicolumn{6}{|c|}{ APTITUDES } & \multirow{3}{*}{$\mathbf{P}$} \\
\hline & \multicolumn{2}{|c|}{ INDIFERENTES } & \multicolumn{2}{|c|}{ DESFAVORABLES } & \multicolumn{2}{|c|}{ FAVORABLES } & \\
\hline & $\mathbf{N}^{\circ}$ & $\%$ & $\mathbf{N}^{\circ}$ & $\%$ & $\mathbf{N}^{\circ}$ & $\%$ & \\
\hline Bajo & 8 & 40.0 & 14 & 21.9 & 3 & 11.5 & 0.247 \\
\hline Medio & 7 & 35.0 & 32 & 50.0 & 15 & 57.7 & \\
\hline Alto & 5 & 25.0 & 18 & 28.1 & 8 & 30.8 & \\
\hline Total & 25 & 100.0 & 54 & 100.0 & 31 & 100.0 & \\
\hline
\end{tabular}


Las aptitudes desfavorables se presentaban en pacientes diabéticos con nivel de conocimientos medio-bajo (50\% y $21.9 \%$ ), pero entre los que tenían aptitudes favorables pocos tenían un bajo nivel de conocimientos (11.5\%). No hay asociación estadísticamente entre las aptitudes y el nivel de conocimientos ( $p>0.05)$ (Tabla $\left.N^{\circ} 3\right)$

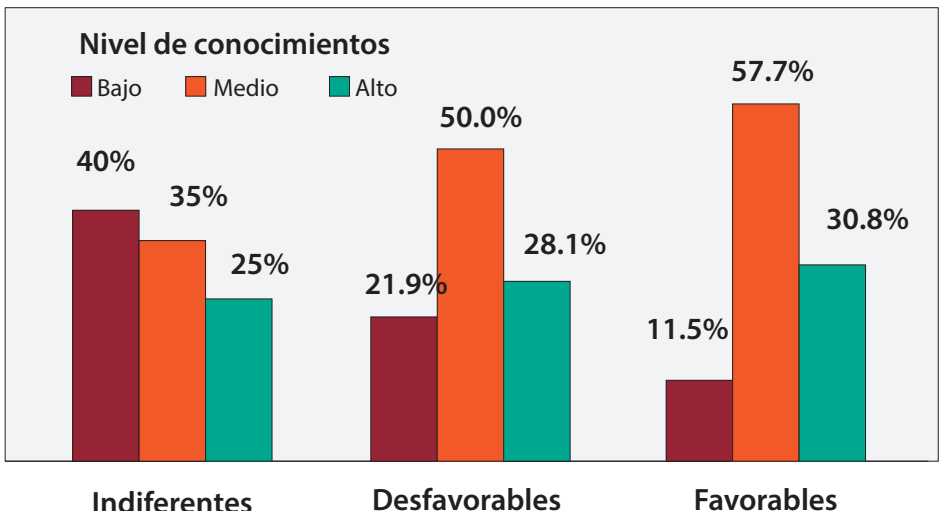

Gráfico 3. Aptitudes y nivel de conocimientos de pacientes diabéticos Servicio de Endocrinología del Hospital de la Policía Nacional del Perú Luis N. Sáenz, 2016.

El bajo nivel de conocimientos predomina en los pacientes diabéticos con aptitudes indiferentes y desfavorables. (Figura 3).

Tabla 4. Asociación entre nivel de conocimientos de complicaciones crónicas de la DM2 y las características de los pacientes diabéticos. Servicio de endocrinología del hospital de la Policía Nacional del Perú "Luis N. Sáenz, 2016".

\begin{tabular}{|c|c|c|c|c|c|c|c|}
\hline & \multicolumn{6}{|c|}{ NIVEL DE CONOCIMIENTOS } & \multirow[t]{3}{*}{$\mathbf{P}$} \\
\hline & \multicolumn{2}{|c|}{ BAJO } & \multicolumn{2}{|c|}{ MEDIO } & \multicolumn{2}{|c|}{ ALTO } & \\
\hline & $\mathbf{N}^{\circ}$ & $\%$ & $\mathbf{N}^{\circ}$ & $\%$ & $\mathbf{N}^{\circ}$ & $\%$ & \\
\hline \multicolumn{8}{|l|}{ Grupos etáreos } \\
\hline 35-39 años & 1 & 4.0 & 4 & 7.4 & 1 & 3.2 & \\
\hline 40-49 años & 7 & 28.0 & 21 & 38.9 & 16 & 51.6 & 0.314 \\
\hline 50-59 años & 11 & 44.0 & 24 & 44.4 & 12 & 38.7 & \\
\hline 60-69 años & 6 & 24.0 & 5 & 9.3 & 2 & 6.5 & \\
\hline \multicolumn{8}{|l|}{ Sexo } \\
\hline Masculino & 5 & 20.0 & 11 & 20.4 & 12 & 38.7 & 0.135 \\
\hline Femenino & 20 & 80.0 & 43 & 79.6 & 19 & 61.3 & \\
\hline \multicolumn{8}{|l|}{ Nivel de instrucción } \\
\hline Primaria & 4 & 16.0 & 6 & 11.1 & 1 & 3.2 & 0.577 \\
\hline Secundaria & 7 & 28.0 & 18 & 33.3 & 12 & 38.7 & \\
\hline Superior & 14 & 56.0 & 30 & 55.6 & 18 & 58.1 & \\
\hline \multicolumn{8}{|l|}{ Tiempo de enfermedad } \\
\hline 1-5 años & 15 & 60.0 & 46 & 85.2 & 22 & 71.0 & 0.066 \\
\hline 6-10 años & 7 & 28.0 & 7 & 13.0 & 7 & 22.6 & \\
\hline 11-15 años & 1 & 4.0 & 1 & 1.9 & 2 & 6.5 & \\
\hline 16-20 años & 2 & 8.0 & 0 & 0.0 & 0 & 0.0 & \\
\hline \multicolumn{8}{|l|}{ Tratamiento farmacológico } \\
\hline Insulina e hipoglicemiantes orales & 11 & 44.0 & 17 & 31.5 & 7 & 22.6 & 0.024 \\
\hline Hipoglicemiantes orales & 7 & 28.0 & 20 & 37.0 & 3 & 9.7 & \\
\hline Insulina & 7 & 28.0 & 17 & 31.5 & 21 & 67.7 & \\
\hline \multicolumn{8}{|c|}{ Adherencia al tratamiento farmacológico } \\
\hline Buena & 17 & 68.0 & 33 & 61.1 & 24 & 77.4 & 0.303 \\
\hline Mala & 8 & 32.0 & 21 & 38.9 & 7 & 22.6 & \\
\hline Total & 25 & 100.0 & 54 & 100.0 & 31 & 100.0 & \\
\hline
\end{tabular}


Al estratificar el nivel de conocimientos según las características de los pacientes diabéticos se evidenció que los que tenían nivel bajo eran de 50-59 años (44\%), sexo femenino (80\%), con instrucción de nivel superior (56\%), un tiempo de enfermedad de 1-5 años (60\%), recibían como tratamiento farmacológico insulina + hipoglicemiantes orales (44\%) y mala adherencia farmacológica (35\%). En tanto que, los diabéticos con conocimientos en nivel alto eran de 4049 años (51.6\%), sexo femenino (61.3\%), instrucción secundaria (58.1\%), tiempo de enfermedad de DM2 de 1-5 años (71\%), tratamiento farmacológico con insulina (67.7\%) y mala adherencia farmacológica (22.6\%). Se evidenció asociación estadísticamente significativa sólo con tratamiento farmacológico de la DM2 ( $p<0.05)$. (Tabla 4)

Tabla 5. Asociación entre nivel de aptitudes desfavorables sobre las complicaciones crónicas de la DM2 y las características de los pacientes diabéticos. Servicio de endocrinología del hospital de la Policía Nacional del Perú "Luis N. Sáenz, 2016".

\begin{tabular}{|c|c|c|c|c|c|c|c|}
\hline & \multicolumn{6}{|c|}{ NIVEL DE CONOCIMIENTOS } & \multirow[t]{3}{*}{$\mathbf{P}$} \\
\hline & \multicolumn{2}{|c|}{ BAJO } & \multicolumn{2}{|c|}{ MEDIO } & \multicolumn{2}{|c|}{ ALTO } & \\
\hline & $\mathbf{N}^{\circ}$ & $\%$ & $\mathbf{N}^{\circ}$ & $\%$ & $\mathbf{N}^{\circ}$ & $\%$ & \\
\hline \multicolumn{8}{|l|}{ Grupos etáreos } \\
\hline 35-39 años & 0 & 0.0 & 5 & 7.8 & 1 & 3.8 & 0.072 \\
\hline 40-49 años & 7 & 35.0 & 28 & 43.8 & 9 & 34.6 & \\
\hline 50-59 años & 7 & 35.0 & 25 & 39.1 & 15 & 57.7 & \\
\hline 60-69 años & 6 & 30.0 & 6 & 9.4 & 1 & 3.8 & \\
\hline \multicolumn{8}{|l|}{ Sexo } \\
\hline Masculino & 2 & 10.0 & 19 & 29.7 & 7 & 26.9 & 0.207 \\
\hline Femenino & 18 & 90.0 & 45 & 70.3 & 19 & 73.1 & \\
\hline \multicolumn{8}{|l|}{ Nivel de instrucción } \\
\hline Primaria & 4 & 20.0 & 6 & 9.4 & 1 & 3.8 & 0.280 \\
\hline Secundaria & 5 & 25.0 & 20 & 31.3 & 12 & 46.2 & \\
\hline Superior & 11 & 55.0 & 38 & 59.4 & 13 & 50.0 & \\
\hline \multicolumn{8}{|l|}{ Tiempo de enfermedad } \\
\hline 1-5 años & 12 & 60.0 & 52 & 81.3 & 19 & 73.1 & 0.055 \\
\hline 6-10 años & 4 & 20.0 & 11 & 17.2 & 6 & 23.1 & \\
\hline 11-15 años & 3 & 15.0 & 0 & 0.0 & 1 & 3.8 & \\
\hline 16-20 años & 1 & 5.0 & 1 & 1.6 & 0 & 0.0 & \\
\hline \multicolumn{8}{|l|}{ Tratamiento farmacológico } \\
\hline Insulina e hipoglicemiantes orales & 11 & 55.0 & 29 & 45.3 & 9 & 34.6 & 0.727 \\
\hline Hipoglicemiantes orales & 5 & 25.0 & 19 & 29.7 & 10 & 38.5 & \\
\hline Insulina & 4 & 20.0 & 16 & 25.0 & 7 & 26.9 & \\
\hline \multicolumn{8}{|c|}{ Adherencia al tratamiento farmacológico } \\
\hline Buena & 13 & 65.0 & 43 & 67.2 & 18 & 69.2 & 0.955 \\
\hline Mala & 7 & 35.0 & 21 & 32.8 & 8 & 30.8 & \\
\hline Total & 20 & 100.0 & 64 & 100.0 & 26 & 100.0 & \\
\hline
\end{tabular}

Al estratificar el nivel de aptitudes según las características de los pacientes diabéticos se evidenció que los que tenían aptitudes desfavorables eran de 40-49 años (43.8\%), sexo femenino (70.3\%), con instrucción de nivel superior (59.4\%), un tiempo de enfermedad de 1-5 años (81.3\%), recibían como tratamiento farmacológico insulina + hipoglicemiantes orales (45.3\%) y mala adherencia farmacológica (32.8\%). Los diabéticos con aptitud favorable tenían
50-59 años (43.8\%), sexo femenino (73.1\%), con instrucción de nivel superior (50\%), un tiempo de enfermedad de 1-5 años (73.1\%), recibían como tratamiento farmacológico hipoglicemiantes orales (38.5\%) y mala adherencia farmacológica (30.8\%). No se encontró asociación estadísticamente significativa entre nivel de aptitudes con ninguna característica de los pacientes diabéticos. (Tabla 5) 


\section{DISCUSIÓN}

En el presente estudio se encuestaron a 110 pacientes cuya edad media fue $49.85 \pm 6.97$ años, siendo predominante de $50-59$ años (42.7\%) similar al estudio de Morales $^{12}$ en Nicaragua, Aguilar y Espinoza ${ }^{16}$ en Bolivia ( $67 \%$ y $80 \%$ eran $>51$ años respectivamente), pero las edades medias fueron superiores a la de los pacientes del Hospital Loayza evaluados por Yance (edad media 44.5+-16.6 años) e inferiores a la serie de Mendizabal ${ }^{18}$ en el Hospital Nacional Carrión del Callao (edad media de 55.6 años) y a la de Untiveros ${ }^{21}$ en el Hospital Nacional Dos de Mayo (64.56 \pm 11.61 años); predominó el sexo femenino (74.5\%) al igual que reportan Morales ${ }^{12}$ (84\%), Aguilar y Espinoza16 (71\%), Yance $^{14}(71 \%)$, Mendizabal $^{18}(70.8 \%)$ y Untiveros ${ }^{21}$ (56.4\%); se encontró que los diabéticos de la serie tenían mayormente instrucción superior (56.4\%) similar a la serie de Yance (53\%) pero contrario a la de Mendizabal ${ }^{18}$ en cuya serie predominaron los de menor nivel educativo.

Respecto al tiempo de enfermedad de DM2 el $75.5 \%$ de nuestra serie tenía de 1-5 años, aunque la serie de Yance $^{14}$ y Morales $^{12}$ presentaban predominio de este mismo periodo de tiempo, pero con menores porcentajes ( $54 \%$ y $56 \%$ respectivamente). Se confirma lo descrito por la literatura, existe un incremento epidemiológico de DM2 y la edad de aparición de la enfermedad en poblaciones cada vez más jóvenes y económicamente activas 24,26,32.

El tratamiento farmacológico más usado era la combinación insulina + hipoglicemiantes orales (44.5\%), aunque otras series nacionales el uso de hipoglicemiantes orales fue predominante como reporta Untiveros ${ }^{21}$ (68.1\% hipoglicemiantes orales vs. $11.7 \%$ insulina).

El $67.3 \%$ de nuestra serie tenía buena adherencia al tratamiento farmacológico y un $32.7 \%$ mala adherencia. Estas características son similares a la serie de Molina ${ }^{19}$ que en el Hospital Nacional Dos de Mayo encontró una buena adherencia en el $68.9 \%$. Estos datos, están dentro del rango descrito por la literatura médica, es decir, que entre un 20 a 40\% de los pacientes diabéticos tienen una mala adherencia al tratamiento farmacológico, el cual es mayor al uso de la insulinoterapia.

Aproximadamente la mitad de los encuestados tenían conocimientos de las complicaciones crónicas de la DM2 en un nivel medio o regular. Sólo un $28.2 \%$ tenían conocimientos en un nivel alto, estos resultados son similares a los reportados por Yance ${ }^{14}$ en diabéticos del Hospital Loayza, donde el nivel de conocimientos medio fue $64 \%$ y presentó menor porcentaje del nivel malo que nuestra serie ( $14 \%$ vs. $22.7 \%$ respectivamente). Esto es contrario a lo que esperábamos encontrar ya que estos pacientes están dentro de un programa de diabetes, donde además de la consulta médica tienen actividades de promoción y prevención de la salud, es decir, un mayor número de diabéticos deberían tener un nivel de conocimientos alto, aunque, el estudio no tiene por finalidad identificar los factores que están involucrados, se puede intuir que existe responsabilidad compartida entre el personal médico y el paciente-familiar ${ }^{28}$.

La investigación permite apreciar algunas diferencias entre los diabéticos con alto y bajo nivel de conocimientos de las complicaciones crónicas de la DM2, así, al observar la edad, los mayores de 50 años tienden a tener nivel bajo, en tanto que los menores de 50 años tienen mayor nivel de conocimientos. Respecto al sexo, las mujeres predominan en porcentaje sobre los varones en todos los niveles del conocimiento, pero se observa que la proporción de varones es mayor cuanto mayor es el nivel de conocimientos. Se demostró una asociación estadística significativa entre nivel de conocimientos y tratamiento farmacológico, así, los que tenían nivel alto mayormente eran pacientes que usaban insulina en tanto el nivel bajo se correspondió con mayor proporción de pacientes con la combinación de insulina + hipoglicemiantes orales $(p<0.05)$. Se evidencia que, a mayor nivel de conocimientos, hay mayor proporción de pacientes con buena adherencia al tratamiento farmacológico, aunque no tuvo significancia estadística para la serie ( $p>0.05)$, No se observa mayor diferencia en el nivel de conocimientos con el grado de instrucción y tiempo de enfermedad del paciente diabético.

La investigación demostró una mayor proporción de pacientes diabéticos con aptitudes desfavorables (58.2\%) que aptitudes favorables (23.6\%), hallazgo similar a lo reportado por Yance $^{14}$ que en su serie encontró aptitudes desfavorables en el 53\% y favorables en el $37 \%$. Estos datos son contrarios a lo esperado si tomamos en cuenta el estudio de Tello ${ }^{13}$ que encontró mayor porcentaje de aptitudes favorables en trabajadores sanos (57\%), los que no reciben mayor información sobre la enfermedad diabética, pero que tienen una mejor aptitud para el autocuidado. Por lo que, las estrategias de intervención no sólo deben abarcar conocimientos, sino, la adopción de cambios actitudinales que permitan lograr los objetivos metabólicos y la práctica de medidas preventivo 
promocionales para evitar o disminuir la progresión de las complicaciones crónicas de la DM2 25-28-32.

\section{CONCLUSIÓN}

La investigación encontró que los pacientes diabéticos aún no han alcanzado los objetivos del Programa de Diabetes del Servicio de Endocrinología del Hospital de la Policía Nacional Luis N. Sáenz, respecto a la adquisición de conocimientos y logro de cambios en la aptitud para el autocuidado como estrategia para prevenir las complicaciones crónicas de la diabetes mellitus 2, tienen mayormente un nivel de conocimientos medio y aptitudes desfavorables para las complicaciones crónicas de la diabetes mellitus 2 .

La investigación no encontró en los pacientes diabéticos encuestados una relación entre nivel de conocimientos y nivel de aptitudes pero si evidencia que existe asociación estadísticamente significativa entre el nivel de conocimientos y el tratamiento farmacológico de la diabetes mellitus 2 .

\section{RECOMENDACIONES}

Las autoridades del Ministerio de Salud y del Hospital de la Policía Nacional del Perú Luis N. Sáenz deberían rediseñar las estrategias educativas del Programa de Diabetes para lograr elevar el nivel de conocimientos sobre complicaciones crónicas de la DM2, así como para modificar conductas y malos hábitos en los pacientes diabéticos que les permitan integrarse al cuidado de su propia salud.

A pesar de que la baja adherencia al tratamiento farmacológico es similar a lo descrito por la literatura.

Se recomienda realizar estudios prospectivos, de diseño causa-efecto para identificar las causas por las que no se han alcanzado mejorar las capacidades intelectuales y actitudinales de los pacientes o estudios de intervención educativa que permitan elevar los conocimientos de los diabéticos sobre su enfermedad, estos estudios valorarán el impacto de las acciones tomadas y de esta forma se podrán realizar los cambios para introducir mejoras de calidad en la atención profesional.

Contribuciones de autoría: El autor Realizó la generación, recolección de información, redacción y aprobación final del artículo original.

Financiamiento: Autofinanciado.

Conflicto de interés: El autor declara no tener conflicto de interés en la publicación de este artículo.

Recibido: 21 de Agosto del 2018

Aprobado: 19 de diciembre del 2018

Correspondencia: Aldo Juvenal Calderon Rivera

Dirección: Psje San Francisco 135-Los Laureles Chorrillos

Teléfono: +51991887134

Correo:ajcalderonrivera@gmail.com

\section{REFERENCIAS BIBLIOGRÁFICAS}

1.Barceló A. La diabetes en las Américas. (en línea) Boletín Epidemiológico. 2001 ene 2008];22(2). (citado 11 de mayo de 2016) Disponible en: http:// www.ops-omsorg/spanish/sha/be_v22n2-diabetes.htm

2. Organización Mundial de la Salud. 10 datos sobre la carga mundial de morbilidad.(en línea) OMS; 2008. (citado 6 de mayo de 2016). Disponible en: http://www.who.int/features/factfiles/global_burden/es/index.html

3. Ministerio de Salud del Perú (MINSA). Tomemos control de la diabetes ¡Ya!. (en línea) Lima, versión electrónica, 2010. (citado 10 de abril de 2016). Disponible en: http://www.minsa.gob.pe/portada/Especiales/2010/ diabetes/queesladiabetes.asp

4. Gayoso D. S, Guerola O. MV, Pinto S. CA, Rivera M. AM. Incidencia de complicaciones tardias en pacientes con diabetes mellitus. (en línea) Universidad Católica de Santa María, Arequipa. (citado 22 de abril de 2016). Disponible en: http://www.ucsm.edu.pe/ciemucsm/pages/t-dbc. htm

5. Muro-L. EMDS, Jiménez-V.MM. Aptitud clínica para atender complicaciones tardías de la diabetes. (en línea) Rev Med Inst Mex Seguro Soc 2009; 47 (2): 141-146. (citado 28 de abril de 2016). Disponible en: http://edumed.imss.

gob.mx/edumed/rev_med/pdf/gra_art/A254.pdf

6. Seclén S, Rosas M, Arias A, Huayta E. Prevalence of type 2 diabetes in peru: First-wave prevalence report from PERUDiab, a population-based threewave longitudinal study. in press. 2015.

7. Instituto Nacional de Estadística e Informática. Resultados de la
Encuesta Demográfica y de Salud Familiar (ENDES 2013). Lima: Instituto Nacional de Estadística e Informática (INEI), Mayo 2014.

8. Hinojosa M. C, Gonzáles E. Prevalencia de los factores de riesgo y de otras enfermedades en el paciente diabético hospitalizado. Endocrinol Nutr 2002; 49(5): 136-9.

9. Sandeep V, Hayward R. Treatment of hypertension in type 2 diabetes mellitus: Blood Pressure Goals, Choice of Agents, and Setting Priorities in Diabetes Care. Ann Intern Med 2003; 2138: 593-602.

10. Organización Mundial de la Salud. Estadísticas Sanitarias Mundiales 2012. Clasificación. NLM: WA 900.1 OMS; 2011. (citado el 15 abril 2016). Disponible en: http://www.who.int/gho/publications/world_health statistics/ES_WHS2012_Full.pdf

11. Organización Panamericana de la Salud (OPS). Enfermedades no transmisibles en las Américas - Indicadores básicos 2011. (en línea). 2011. (citado 5 de mayo de 2016). Disponible en: www.paho.org

12. Morales V. J. Conocimientos, actitudes y prácticas de los pacientes diabéticos, Programa dispensarizados en los 6 centro de salud del SILAIS Estelí- Nicaragua, Mayo a Junio del 2007. Universidad Nacional Autonoma De Nicaragua Centro de investigaciones y estudios de la salud Maestria en salud pública 2005 - 2007. (en línea) Tesis para optar al título de Master en Salud Pública. OCOTAL,

NUEVA SEGOVIA 2007. . (citado 8 de mayo de 2016). Disponible en: http:// cedoc.cies.edu.ni/digitaliza/t381/doc-contenido.pdf

13. Tello V. M. Conocimientos, actitudes y prácticas de las medidas 
preventivas de la diabetes mellitus tipo 2 en los trabajadores mayores de 35 años de los centros de aplicación: productos unión y editorial imprenta Unión De La Universidad Peruana Unión - Lima, 2011. (en línea) Dirección General de Investigación I Congreso Nacional De Investigación - Universidad Peruana Unión. . (citado 10 de mayo de 2016). Disponible en: http://papiros.upeu.edu.pe/bitstream/handle/ 123456789/109/ CSS16Articulo.pdf? sequence $=1$

14. Yance S.LH. Nivel de conocimientos y aptitudes sobre complicaciones de la diabetes mellitus 2 en el Servicio de Endocrinología del Hospital Nacional Arzobispo Loayza, Agosto-Setiembre 2013. Tesis de grado, Universidad Nacional Federico Villareal. 2014: 11-37

15. Steed L, Cooke D, Newman S. A systematic review of psychosocial outcomes following education, self-management and psychosocail interventions in diabetes mellitus. Patient Educ Counseling 2003;51(1):5-15.

16. Aguilar E. J, Espinoza D. E. Evaluación del conocimiento y práctica dietetica en pacientes diabéticos tipo 2. Gac Med Bol. (en línea). 2006; 29 (1):17-20 (citado 11 de mayo de 2016). Disponible en: http://www.scielo.org.bo/scielo.php? script=sci_arttext\&pid=S1012 29662006000100004\&lng=es\&nrm=iso

17. Sabag R. E. Complicaciones crónicas de la diabetes mellitus. Prevalencia en una unidad de

medicina familiar. (en línea) Rev Med Inst Mex Seguro Soc 2006; 44 (5): 415-421. (citado 3 de mayo de 2016). Disponible en: http://www. medigraphic com/pdfs/imss/im-2006/im065e.pdf

18. Mendizábal T, Navarro N, Ramírez A, Cervera M, Estrada E, Ruiz I. Características sociodemográficas y clínicas de pacientes con diabetes tipo 2 y microangiopatías. (en línea) An Fac med. 2010; 71(1) :7-12. (citado 10 de mayo de 2016). Disponible en: http://www.scielo.org.pe/ pdf/afm/v71n1/a02v71n1.pdf

19. Molina G.YR. Adherencia al tratamiento y su relación con la calidad de vida de los pacientes con diabetes mellitus tipo 2 que asisten al Programa de Diabetes del Hospital Nacional "Dos de Mayo" : enero-febrero 2008. (en línea) Tesis para optar el título profesional de Licenciada en Enfermería. Universidad Nacional Mayor de San Marcos, Lima, Perú (2008). (citado 11 de mayo de 2016). Disponible en: http://www.cybertesis.edu.pe/ sisbib/2008/molina_gy/html/index-frames.html

20. Hidalgo C. EV. Medidas de autocuidado que realizan los pacientes diabéticos. Factores socioculturales que favorecen o limitan su cumplimiento en los pacientes que asisten al Programa de Diabetes del Hospital Nacional Dos de Mayo, octubre-diciembre 2005. (en línea) Tesis Para optar el Título profesional de licenciada en enfermería. Universidad Nacional Mayor de San Marcos, Lima, Perú (2005). (citado 5 de mayo de 2016). Disponible en: http://www.cybertesis.edu.pe/ sisbib/2005/ hidalgo_ce/html/index-frames.htm

21. Untiveros M. CF, Núñez Ch. O, Tapia Z. LM, Tapia Z. GG.

Complicaciones tardías en diabetes mellitus tipo 2 en el Hospital II Essalud - Cañete. (en línea) Rev Med Hered 2004:15(2): 12-7. (citado 5 de mayo de 2016). Disponible en: http://www.scielo.org.pe/pdf/rmh/v15n2/ v15n2ao1.pdf

22. Molero T. GR. Complicaciones tardias en pacientes con diabetes mellitus del hospital I EsSalud Quillabamba - 2003. (en línea) SITUA - Revista Semestral de la Facultad de Medicina Humana - UNSAAC. (citado 5 de mayo de 2016). Disponible en: http://sisbib.unmsm.edu.pe/ bvrevistas/situa/2003_n22/EnPDF /complic_tardias.pdf

23. American Diabetes Association. Third party reimbursement for diabetes care, self management education, and supplies. Diabetes Care. 24 (Suppl. 1): S120-S121.. 2001

24. Elaboración de la Encuesta de Salud Integral - Atención primaria de salud y participación comunitaria. (citado 3 de mayo de 2016). Disponible en: http://es.scribd.com/doc/23579911/Encuesta-de-salud-integral-enatencion-primaria

25. Monja Y, JC. Características epidemiológicas clínicas y metabólicas de la nefropatía en pacientes diabéticos tipo 2, hospitalizados en el Servicio de Medicina Interna No 3 del HNGAI (2004). (en línea) Universidad Nacional Mayor de San Marcos, Lima, Perú. (citado 10 de mayo de 2016). Disponible en: http://www.cybertesis.edu.pe/sisbib/2004/monja_yj/ html/index-frames.html

26. Choi BC, Corber SJ, McQueen DV, Bonita R, Zevallos JC, Douglas KA, et al. Enhancing national capacity in chronic disease surveillance in

the Americas. Pan Am J Public Health. 2005;17(2):130-41.

27. Scanlon PH. Why do patients still require surgery for the late complications of proliferative diabetic retinopathy? Eye. 2010;24(3):435-41.

28. The Diabetes Control and Complications Trial Research Group. The effect of intensive treatment of diabetes on the development and progression of long-term complications in insulin-dependent diabetes mellitus. N Engl J Med. 1993; 329:977-86

29. Qi I, Rimm E, Liu S, Rifai N, Hu FB. Dietary glycemic index, glycemic load, cereal fiber, and plasma adiponectin concentration in diabetic men Diabetes Care. 2005;28:1022-8.

30. Bantle JP, Wylie-Rosett J, Albright AL, Apovian CM, Clark NG, Franz MJ, et al. Nutrition recommendations and inter ventions for diabetes: a position statement of the American Diabetes Association. Diabetes Care. 2007;30(Suppl 1):S48-65

31. Litwak L, Calella P, Chetkoff A, Cutuli H, Katz S, Lúquez C. Estado metabólico y características de la subpoblación de pacientes argentinos que participaron del estudio A1chieve. Rev Soc Arg de Diabetes 2012; 46: 49-56.

32. Fracchini M. Cambio de conductas en tratamientos de larga duración Relación médico-paciente. Medicina (B Aires) 2004; 64: 550-4.

33. García R, Suárez R. Resultados de un seguimiento educativo a personas con diabetes mellitus tipo 2 y sobrepeso u obesidad. (en línea) Rev Cubana Endocrinol. 2003; 14(3): [aprox. 9 p.]. (citado 10 de mayo de 2016) Disponible en: http://bvs.sld.cu/revistas/end/vol14_3_03/end04303.htm

\section{Indizado en: latindex}

http://www.latindex.org/latindex/ficha?folio=14280

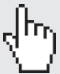

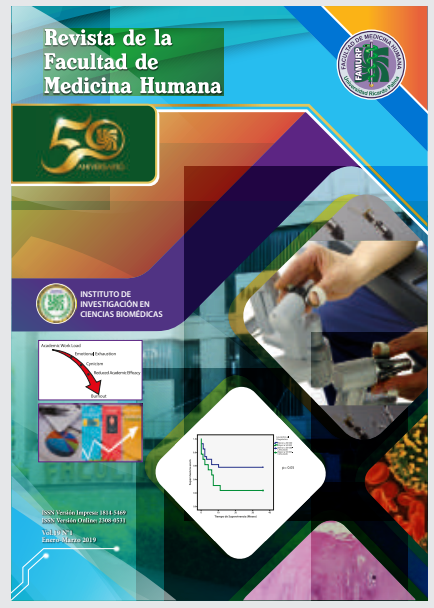

